\title{
Improving Effectiveness of Student Learning Experiences during Clinical Ro- tations at Rwanda Military Hospital (RMH)
}

\author{
Sophie Kairanga ${ }^{1}$, Josette Niyokindi ${ }^{1}$, Nanyombi Lubimbi ${ }^{1}$, Kathryn Norgang ${ }^{1}$, Josee Uwambaye ${ }^{1}$ \\ ${ }^{1}$ Rwanda Military Hospital, Rwanda
}

\section{Background}

In Rwanda there currently exists a shortage of clinical nursing instructors which impacts the clinical supervision of the nursing and midwifery students. One of the major barriers to learning includes a history of student absenteeism. The Human Resources for Health (HRH) clinical mentors together with their Rwandan faculty counterparts identified the need to create a favorable and effective learning environment at the Rwanda Military Hospital.

\section{Methods}

On the first day, during orientation, the expectations of the students were identified and discussed. These expectations included collaboration with the clinical instructors, ward staff nurses, and the HRH clinical mentors. The ward managers facilitated structured learning by assisting the students in the identification of patient assignments using the curriculum learning objectives as a guide, thus facilitating appropriate selection of patients to meet these objectives. Throughout all clinical rotations with the students, the HRH clinical mentors, in collaboration with clinical faculty, were present at all times. The faculty presence allowed ongoing evaluation of care modalities and of learning in general, with interactive constructive feedback with the students. Post-conference was provided once to twice a week focusing on evidenced-based practice and innovations in care through the inclusion of case reviews and practice in critical assessment skills.

\section{Results}

The students were observed to be more confident in knowledge and skills during clinical rotations. Clinical faculty evaluations by students highlighted the students' satisfaction with the process and positive recommendations were made for the continuation of the structured learning environment.

\section{Conclusion}

Based on the approval of both students and clinical instructors, we have identified the ongoing need for a structured learning environment during clinical rotations at Rwanda Military Hospital. We acknowledge that evidence-based practice and engaging instructions are necessary to effectively address the learning needs of nursing and midwifery students throughout the country.

Key words: clinical rotation, structured learning, critical thinking skills, post-conference, Rwanda 